\title{
The emerging picture of the mitochondrial protein import complexes of Amoebozoa supergroup
}

\author{
Małgorzata Wojtkowska ${ }^{1 *}$ (D), Dorota Buczek ${ }^{1,2}$, Yutaka Suzuki ${ }^{3}$, Victoria Shabardina ${ }^{2}$, Wojciech Makałowski ${ }^{2}$ \\ and Hanna Kmita ${ }^{1}$
}

\begin{abstract}
Background: The existence of mitochondria-related organelles (MROs) is proposed for eukaryotic organisms. The Amoebozoa includes some organisms that are known to have mitosomes but also organisms that have aerobic mitochondria. However, the mitochondrial protein apparatus of this supergroup remains largely unsampled, except for the mitochondrial outer membrane import complexes studied recently. Therefore, in this study we investigated the mitochondrial inner membrane and intermembrane space complexes, using the available genome and transcriptome sequences.
\end{abstract}

Results: When compared with the canonical cognate complexes described for the yeast Saccharomyces cerevisiae, amoebozoans with aerobic mitochondria, display lower differences in the number of subunits predicted for these complexes than the mitochondrial outer membrane complexes, although the predicted subunits appear to display different levels of diversity in regard to phylogenetic position and isoform numbers. For the putative mitosome-bearing amoebozoans, the number of predicted subunits suggests the complex elimination distinctly more pronounced than in the case of the outer membrane ones.

Conclusion: The results concern the problem of mitochondrial and mitosome protein import machinery structural variability and the reduction of their complexity within the currently defined supergroup of Amoebozoa. This results are crucial for better understanding of the Amoebozoa taxa of both biomedical and evolutionary importance.

Keywords: Amoebozoa, Mitochondria, Mitosomes, Protein import, TIM22 complex, TIM23 complex, small Tims, MIA complex, PAM complex, OXA complex

\section{Background}

Currently, the division of eukaryotic organisms consists of six large supergroups, namely Chromalveolata, Excavata, Archaeplastida, Rhizaria, Amoebozoa, and Opisthokonta $[1,2]$. As summarized in Fig. 1, the Amoebozoa, regarded as a sister clade to the Opisthokonta which involves animals and fungi, include some organisms that are known to have mitosomes, such as Entamoeba histolytica, an intestinal pathogen of humans [3, 4], but also organisms that have aerobic mitochondria, such as the slime mold Dictyostelium discoideum, a free-living organism

\footnotetext{
* Correspondence: woytek@amu.edu.pl

${ }^{1}$ Laboratory of Bioenergetics, Institute of Molecular Biology and

Biotechnology, Faculty of Biology, Adam Mickiewicz University, Umultowska

89, 61-614 Poznan, Poland

Full list of author information is available at the end of the article
}

that inhabits soil and compost [5], as well as the amoeba Acanthamoeba castellanii, a soil free-living amoeba also known as a facultative human parasite [6]. The organisms represent different subclades and subdivisions of the Amoebozoa [1]. A. castellanii represents the Lobosa, whereas $D$. discoideum and $E$. histolytica belong to the Conosa and are included in the Mycetozoa and Archamoebae, respectively (Fig. 1). Thus, the Amoebozoa encompass taxa of both biomedical and evolutionary importance, yet their genomic and transcriptomic diversity remains largely unsampled. The same applies to the mitochondrial protein import complexes. Accordingly, the data concerning the subunit organization of the mitochondrial protein import complexes for members of the 


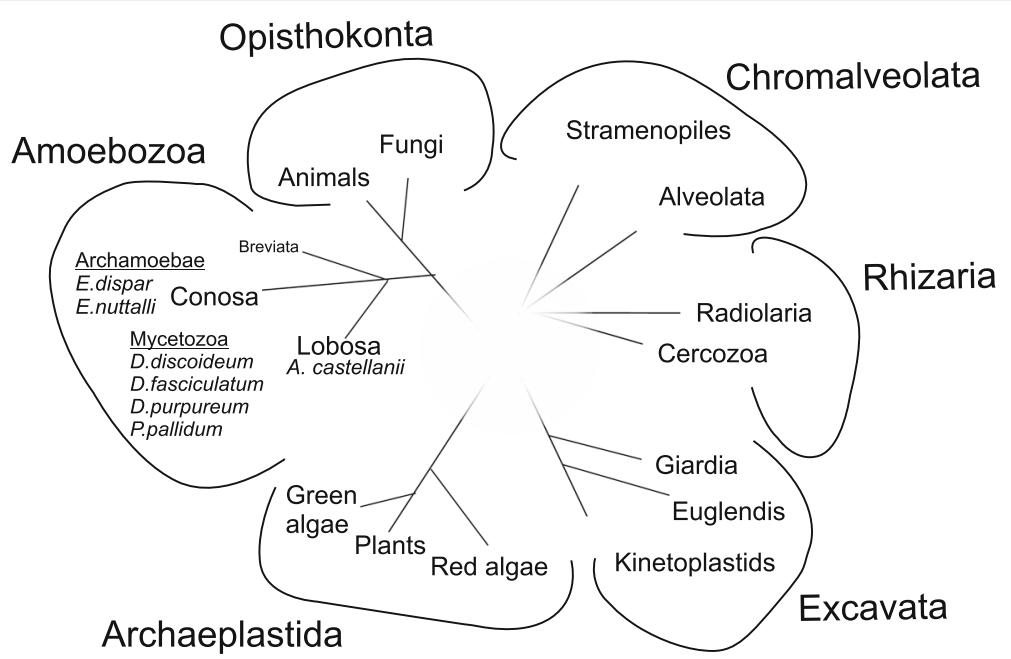

Fig. 1 Schematic representation of the phylogenetic position of the studied organisms in the eukaryotic tree. Based on [1, 2, 46]

same supergroup classified into distinct subclades and divisions are not well known.

Studies of the mitochondrial protein import machinery have revealed that the machinery consists of complexes located in all mitochondrial compartments and have provided information about their organization, function, and interplay. The most commonly used model organism in the studies is the yeast Saccharomyces cerevisiae. Consequently, the S. cerevisiae protein import machinery, schematically shown in Fig. 2, is defined as a canon and applied as a reference model in studies of the machinery of other eukaryotic organisms, including plants and animals [7-9]. The canon includes the presence of at least three complexes in the outer membrane, four in the inner membrane, and three in the intermembrane space. The TOM (translocase of the outer membrane), TOB/SAM (topogenesis of the mitochondrial outer membrane $\beta$-barrel proteins/sorting and assembly machinery) and MIM (mitochondrial import machinery) complexes are located in the outer membrane. The TOM complex represents a general entry gate for most proteins imported into mitochondria, whereas the TOB/ SAM complex and MIM complex enable the insertion of $\beta$-barrel proteins and proteins containing one or more transmembrane-spanning helices into the membrane, respectively. The TIM22 (translocase of the inner membrane 22), TIM23 (translocase of the inner membrane 23), PAM (presequence translocase-associated motor), and OXA (oxidase assembly factor) complexes are located in the inner membrane. The TIM22 and TIM23 complexes mediate the import of precursor proteins with targeting signals located within their sequence and at their $\mathrm{N}$-terminus, respectively. The PAM complex cooperates with the TIM23 complex to drive protein translocation into matrix, whereas the OXA complex participates in the insertion of proteins from the matrix side into the inner membrane. The MIA (mitochondrial intermembrane space assembly) complex as well as small Tim chaperone-like protein complexes (small Tims), i.e. Tim8-Tim13 and Tim9-Tim10-Tim12 complexes, are located in the intermembrane space. The MIA complex is responsible for the import of small intermembrane space proteins with the multiple cysteine residues due to the thiol-disulfide exchange, whereas small Tims associate with the TOB/SAM and TIM22 complexes to protect precursor proteins from misfolding in the intermembrane space.

Available data indicate that the subunit organization of the complexes of animals and plants is more or less similar to those depicted in Fig. 2 for S. cerevisiae (for reviews, see e.g. [7, 9-11] although some new subunits have recently been identified for animals e.g. [10] and plants [11]. Nevertheless, in other eukaryotes, the differences are more pronounced. Consequently, the commonly occurring subunits of the TOM, TOB/SAM, and TIM22 complexes are their channel-forming subunits, Tom40, Sam50/Tob55, and Tim22, respectively, whereas the common presence of Tim50, Tim17, and Tim23 is reported for the TIM23 complex, Erv1 for the MIA complex, and Tim 9 and Tim10 for the small Tim protein complexes [12, 13]. In the case of the MIM and OXA complexes, data are still missing for this kind of analysis. The data concerning the protein import machinery are also strongly limited in mitosome-carrying organisms. As summarized by Heinz and Lithgow [12] as well as by Makiuchi and Nozaki [13], they are available for Cryptosporidium parvum (Chromalveolata), Encephalitozoon cuniculi (Opisthokonta), Giardia intestinalis (Excavata), and E. histolytica (Amoebozoa). The organisms carry Tom40 and Sam50/Tob55, although the 


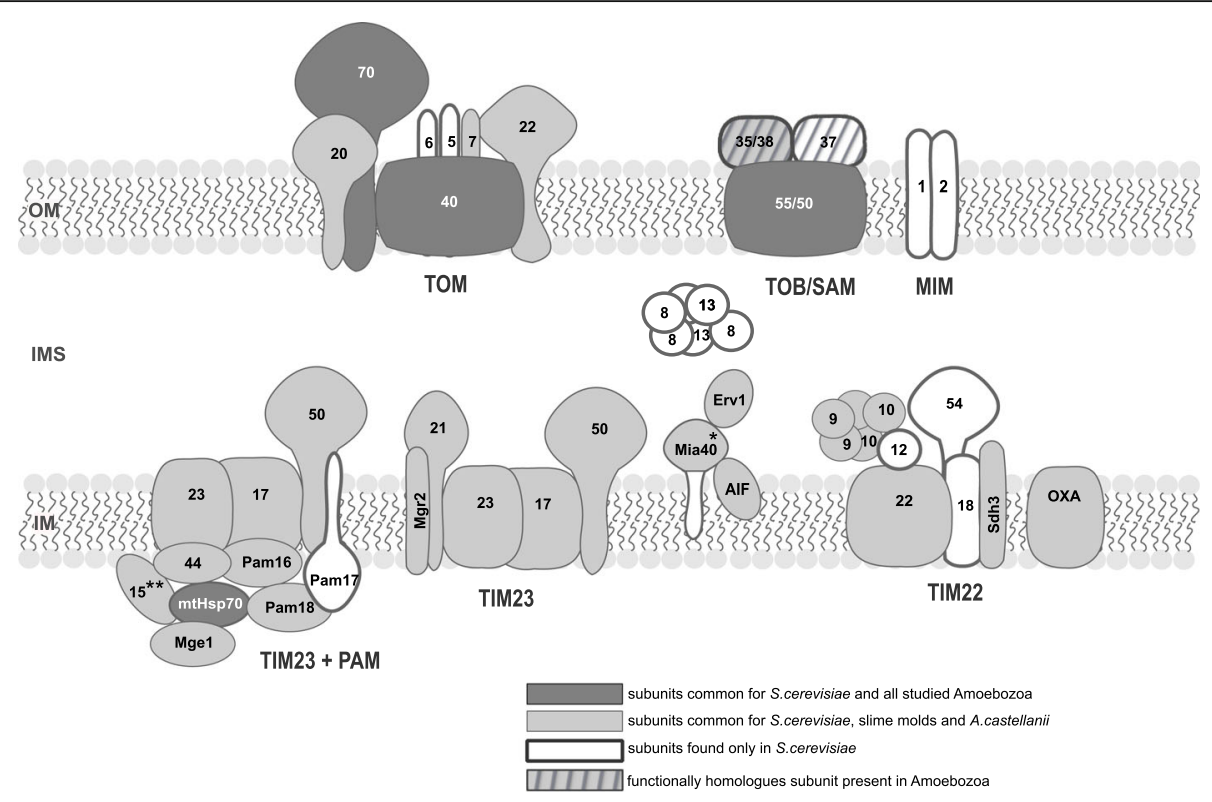

Fig. 2 Schematic representation of the mitochondrial protein import complexes of Saccharomyces cerevisiae and their subunits shared with the studied amoebozoans. The TOM complex contains a channel-forming subunit (Tom40), receptors for precursor proteins (Tom20 and Tom70), an internal receptor, which also maintains the complex architecture (Tom22), and modulators of the complex assembly and stability (Tom5, Tom6, and Tom7). The TOB/SAM complex contains a channel-forming subunit (Tob55/Sam50), which cooperates with Sam35/Tob38 and Sam37/Mas37 in the recognition, transport, and integration of $\beta$-barrel proteins into the membrane. The TIM22 complex is composed of a channel-forming subunit (Tim22) and three modulators of the complex stability and activity, i.e. Tim18, Tim54, and Sdh3. The TIM23 complex consists of Tim50 (which functions as a receptor in the initial stages of precursor translocation), Tim23 and Tim17 (forming a channel within the complex), as well as Tim21 and Mgr2 (suggested to play an important role in the cooperation with the respiratory chain) The absence of Mgr2 enables the TIM23 complex interaction with the PAM complex. The complex consists of $\mathrm{mtHsp70,} \mathrm{Tim44,} \mathrm{and} \mathrm{a} \mathrm{group} \mathrm{of} \mathrm{co-chaperones} \mathrm{and} \mathrm{supporting} \mathrm{proteins,} \mathrm{i.e.}$ Pam16 (Tim16), Pam18 (Tim14), the nucleotide exchange factor Mge1, Pam17 and Tim15. The OXA complex is formed by Oxa1, which is thought to cooperate with its paralog, Oxa2, serving as an auxiliary subunit. The MIA complex consists of two proteins, namely the oxidoreductase Mia40 and sulfhydryl oxidase Erv1. ${ }^{*}$, subunits found only for slime molds; ${ }^{* *}$, a subunit found only for A. castellanii; AlF - apoptosis inducing factor

latter was not detected for G. intestinalis, and only two receptor subunits of the TOM complex, Tom70 and Tom60, were predicted for E. cuniculi and E. histolytica, respectively. Moreover, C. parvum and E. cuniculi have Tim17 and Tim23 (the TIM23 complex) as well as Tim22 (the TIM22 complex), but it is difficult to distinguish the proteins at the level of their sequences. Other subunits of the complexes, Tim50 (the TIM23 complex) and Tim18 (the TIM22 complex), were found for $C$. parvum and E. cuniculi with the exception of G. intestinalis Tim18. In the case of small Tims, only Tim8 and Tim13 seem to be expressed by C. parvum. As far as the PAM complex subunits are concerned, mtHsp70 was found for all the mentioned organisms, Mge1 for C. parvum, G. intestinalis, and E. histolytica, while Tim44 and Pam16 were detected only for C. parvum.

Our previous study, based on searching of the available genome and transcriptome data concerning amoebozoan subunits of the TOM and TOB/SAM complexes, indicated that the complexes may display structural variability in the Amoebozoa lineage and their subunit number reduction, as compared with the corresponding canonical complexes of S. cerevisiae [14]. Accordingly, the predicted number of subunits of the TOM and TOB/SAM complexes were different for A. castellanii, and representatives of slime molds and entamoebas as well as within the slime molds and entamoebas. Importantly, entamoebas are proposed to contain mitosomes and $E$. dispar, and E. nuttalii are now recognized as separate species from the well-known pathogenic E. histolytica and E. invadens, although their pathogenicity is still a matter of debate [15-17]. To study the issue further, we extended our research on the transcriptome and genome analysis of the complexes located in the inner membrane and intermembrane space of the amoebozoan mitochondria. As mentioned above, they include the TIM22, TIM23, PAM, and OXA complexes located in the inner membrane as well as the MIA complex and the complexes of small Tim proteins forming Tim8Tim13, and Tim9-Tim10-Tim12 complexes, all located in the intermembrane space. The predicted subunits indicate that for amoebozoans with aerobic mitochondria, organization variability of the complexes may be less pronounced than in the case of the mitochondrial outer membrane complexes. Nevertheless, the subunits predicted for the inner membrane and the intermembrane 
space complexes display different levels of diversity in regard to phylogenetic position and some of them have isoforms. Moreover, elimination of the complexes in the putative mitosome-bearing amoebozoans is predicted to be much more conspicuous than in the case of the outer membrane complexes. The results appear to be important for discussion on the complex localization and functions as well as their contribution to the protein import and functions of mitochondrial membranes.

\section{Methods}

\section{Species of amoebozoans}

The studied organisms included Acanthamoeba castellanii (Lobosa; Discosea), Dictyostelium discoideum, D. purpureum, D. fasciculatum, and Polysphondylium pallidum (Conosa; Mycetozoa), as well as Entamoeba dispar and E. nuttalli (Conosa; Archamoebae). Additional file 1: Table S1 summarizes data concerning their genome and transcriptome sequences.

Acanthamoeba castellanii strain Neff used in this study was delivered from American type Culture Collection (ATCC) with the number 30010.

\section{Acanthamoeba castellanii cell culture and isolation of total RNA}

Cells of Acanthamoeba castellanii (strain Neff) were cultured at $28{ }^{\circ} \mathrm{C}$, in an axenic environment in the standard medium described by Neff [18] with some modifications: $1.5 \%$ proteoso-peptone, $0.15 \%$ yeast extract, $30 \mathrm{mM}$ $\mathrm{MgCl}_{2}, 30 \mathrm{mM} \mathrm{FeSO}$, $27 \mathrm{mM} \mathrm{CaCl} 2,1.5 \%$ glucose, $2.5 \mathrm{mg} / \mathrm{l}$ vitamin B12, $1 \mathrm{mg} / \mathrm{l}$ vitamin $\mathrm{B} 1$, and $0.2 \mathrm{mg} / \mathrm{l}$ vitamin $\mathrm{H}$. Cells in the trophozoite stage were collected in the intermediary phase after $48 \mathrm{~h}$ and next were frozen in liquid nitrogen and homogenized in TRizol reagent (Invitrogen). Total RNA was isolated according to the manufacturer's instructions (Invitrogen). DNase I was added to eliminate remaining genomic DNA. The absence of DNA was confirmed by PCR and agarose gel electrophoresis.

\section{Acanthamoeba castellanii cDNA preparation, sequencing, and transcriptome assembly}

cDNA was prepared using an mRNA-Seq Sample preparation Kit (Illumina), according to the manufacturer's instructions. Sequencing of the cDNA, i.e. mRNA-Seq of A. castellanii was performed on the HiSeq 2000 platform (Illumina) with 36-bp single-end reads. AC_RNASeq data are available in DDBJ database; the accession number DRA006231 (BioSample number SAMD00097225). The obtained raw reads were subjected to quality control analysis. After removal of poorquality sequences, short reads were assembled using Trinity RNA-Seq [19] with -SS_lib_type F (AC_RNASeq) and min_contig_length 300 .

\section{Prediction of proteins}

To find the best annotated amino acid sequences for subunits of the TIM22, TIM23, PAM, OXA, small Tims and MIA complexes, keyword searches against the NCBI (http://www.ncbi.nlm.nih.gov/) and Pfam (http://pfam.sanger.ac.uk) databases were performed. First, sets of well-known sequences from different species representing various eukaryotic lineages including subunits identified for S. cerevisiae as well as detected specifically for plant or animal mitochondrial protein import complexes (Additional file 1: Table S2) were used as queries in tBLASTn searches [20] against the transcriptome of $A$. castellanii with variable $e$-values (from $10^{-3}$ to 1 ). For the proteins that were not predicted by tBLASTn, a HMMER search based on Hidden Markov Model was performed [21]. In the case of multiple-protein hits, sequences giving the highest coverage of transcripts were selected. To translate the transcripts into protein sequences, the ExPASY server was used [22]. The amino acid sequences of putative proteins were subjected to a BLASTp [20] search in order to compare the sequences with available protein datasets of $A$. castellanii [6]. To find previously un-annotated proteins, a tBLASTn search against the available genome of $A$. castellanii was performed.

Subsequently, proteins predicted for A. castellanii were used in a tBLASTn search against the protein datasets of Dictyostelium purpureum, D. discoideum, D. fasciculatum, Polysphondylium pallidum, Entamoeba dispar, and Entamoeba nuttalli (Additional file 1: Table $\mathrm{S1}$ ). For the proteins that were not predicted by the analysis, the tBLASTn algorithm was used against the available genomes of $D$. discoideum, $D$. purpureum, $D$. fasciculatum, P. pallidum, E. dispar, and E. nuttalii. Finally, reference sequences from various eukaryotic lineages (Additional file 1: Table S2) were used to predict proteins not found by the previously applied methods.

\section{Phylogenetic inference}

Protein sequences were aligned using CLUSTAL W [23] and phylogenetic trees were inferred using the Neighbor-Joining method [24] with the amino acid substitution model using Poisson correction as implemented in MEGA (version 7) software [25, 26]. The data were bootstrapped by 1000 replicates [27].

\section{Results}

We investigated the presence of genes encoding subunits of the mitochondrial protein import complexes located in the intermembrane space and inner membrane using available genome and transcriptome sequences. As summarized in Table S1 (Additional file 1), among the studied amoebozoans the transcriptome data are not available for the slime mold Dictyostelium fasciculatum 
and both the entamoebas, i.e. Entamoeba nuttalli and Entamoeba dispar. Moreover, in the case of the amoeba Acathamoeba castellanii we applied two transcriptome datasets, one already available at GenBank [6] and the second (AC_RNAseq) assembled by us (see Methods). The reason is that we noticed some differences between the transcriptome datasets reflected in amino acid sequences of proteins predicted for the mitochondrial outer membrane [14]. The involvement of transcriptome data supplies more information regarding gene isoforms and gene structure giving more opportunity in prediction process of examined genes.

\section{Subunits predicted for $A$. castellanii}

The application of the available GenBank and AC_RNASeq enabled prediction of $17 \mathrm{~A}$. castellanii possible subunits of mitochondrial protein import complexes located in the intermembrane space and the inner membrane (Table 1; for the complex organization see Fig. 2). Importantly, four of the subunits appeared to have isoforms (Tim9, Tim10, Pam16, and Pam18). All the predicted proteins displayed a high level of amino acid sequence identity to the GenBank data but also some differences were observed when AC_RNASeq was taken into account. The differences are shown in Additional file 1: Figures S1 and S2.

The amino acid sequences predicted for Tim21, Tim44, Erv1 and Oxa1 have been already specifically annotated so the existing accession numbers were added to Table 1. The sequences predicted for Tim9C, Tim10A, Sdh3, Tim17, Tim23, Tim15 and Pam18A have been named specifically (Table 1, underlined letters; Tim15 is under the process of annotation). To check the accuracy of the predicted A. castellanii Tim 9 and Tim10 isoforms the phylogenetic analysis was performed (Fig. 3a). In the obtained phylogenetic tree, two clusters representing Tim 9 and Tim10 families can be easily distinguished. Although bootstrap values were not very high for the internal branches within two clusters, they were separated by the middle branch, which was supported by the bootstrap value of $98 \%$. Thus, the isoforms were predicted properly and they appeared to be orthologs. Accordingly, to estimate amino acid similarity between $A$. castellanii isoforms of Pam16 and Pam18 and their phylogenetic relationships, phylogenetic trees were built (Fig. $3 \mathrm{~b}-\mathrm{c}$ ). The obtained results indicated that the slime mold clades of Pam16 and Pam18 grouped with A. castellanii clades of these proteins. Thus the A. castellanii proteins appeared to be orthologs.

For the rest of the predicted proteins some differences were observed between AC_RNAseq and the GenBank data (Additional file 1: Figure S1). Consequently, we proposed some corrections of sequences predicted for Tim9A, Tim10B, Tim22, Tim50, Mgr2, Pam16A,
Pam16B, and $\mathrm{mtHsp70}$. In the case of the predicted Tim9A, the protein already stored in the GenBank (XP_004340603) was mis-annotated, as it contained only part of the functional domain of Tim9 proteins, and was fused with EF-hand $\mathrm{Ca}^{2+}$-binding domain that was removed from the corrected Tim9A version. The predicted Tim10B, Tim22, and Pam16A were longer than cognate annotated proteins (XP_004336134, AAT66174 and XP_004335678, respectively), whereas Tim50 and Mgr2 were longer than hypothetical proteins (XP_004344840 and XP_004333275, respectively). The protein XP_004368030.1 appeared to be encoded by an erroneously fused gene that was eliminated from the corrected Pam16B version. In the case of predicted mtHsp70, the correction concerned two amino acid substitutions at the C-terminus, namely alanine to serine and leucyne to methionine. Finally, the genes encoding Tim9B, Pam18B, and Mge1 were not present in the GenBank data but were supported by hits found in tBLASTn alignment against AC_RNASeq (Additional file 1: Figure S2).

Summing up, as can be concluded from data shown in Table 1, organization of the A. castellanii TIM23 and PAM complexes may be identical to cognate complexes of $S$. cerevisiae whereas in the case of small Tims, TIM22, OXA and MIA complexes some subunits described for cognate $S$. cerevisiae complexes appear not to be present.

\section{Subunits predicted for slime molds}

Altogether 18 subunits were predicted for the intermembrane space and inner membrane import complexes (Fig. 2) of the studied slime molds, i.e. $D$. discoideum, $D$. fasciculatum, D. purpureum and Polysphondylium pallidum (Table 1). Most of the proteins were identical to sequences stored in the GenBank as specifically annotated or hypothetical proteins (the latters are marked as underlined letters in Table 1). The isoforms were predicted only for Oxa1. To check their accuracy phylogenetic analysis was performed and the obtained phylogenetic tree supported the notion that the slime mold Oxa1A and Oxa1B were paralogs (Fig. 3d). Moreover, for A. castellanii Oxa1 a higher similarity to the slime mold Oxa1B was observed. The only correction concerned P. pallidum Tim9, detected by the genome data analysis. The sequence turned out to be a part of a sequence stored in the GenBank under accession number EFA81367 (Additional file 1: Figure S1B).

Summing up, it appears that organization of the slime mold TIM23, PAM and MIA complexes may be identical to cognate complexes of $S$. cerevisiae whereas in the case of small Tims, TIM22 and OXA complexes some subunits are probably not present. Interestingly, with 


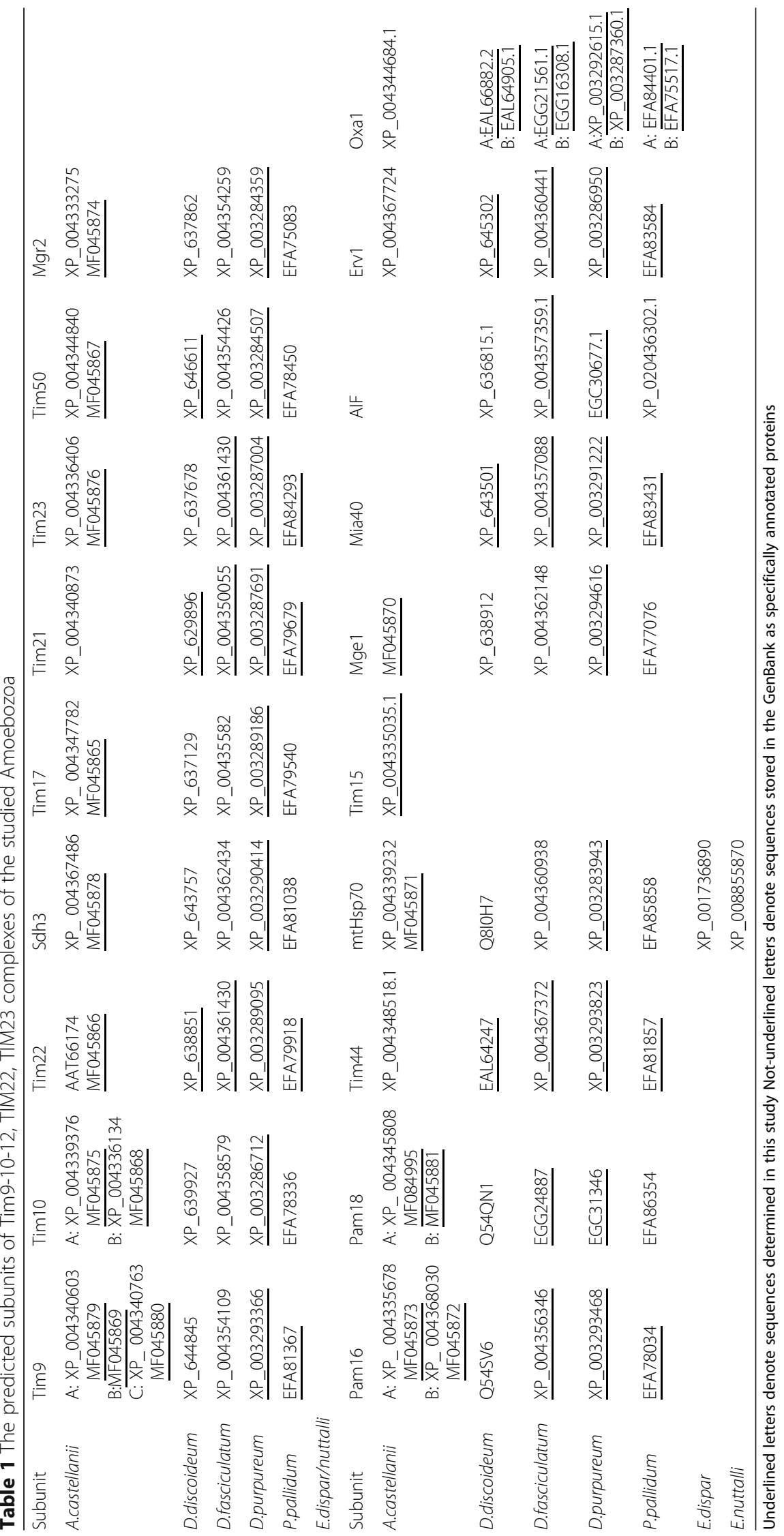




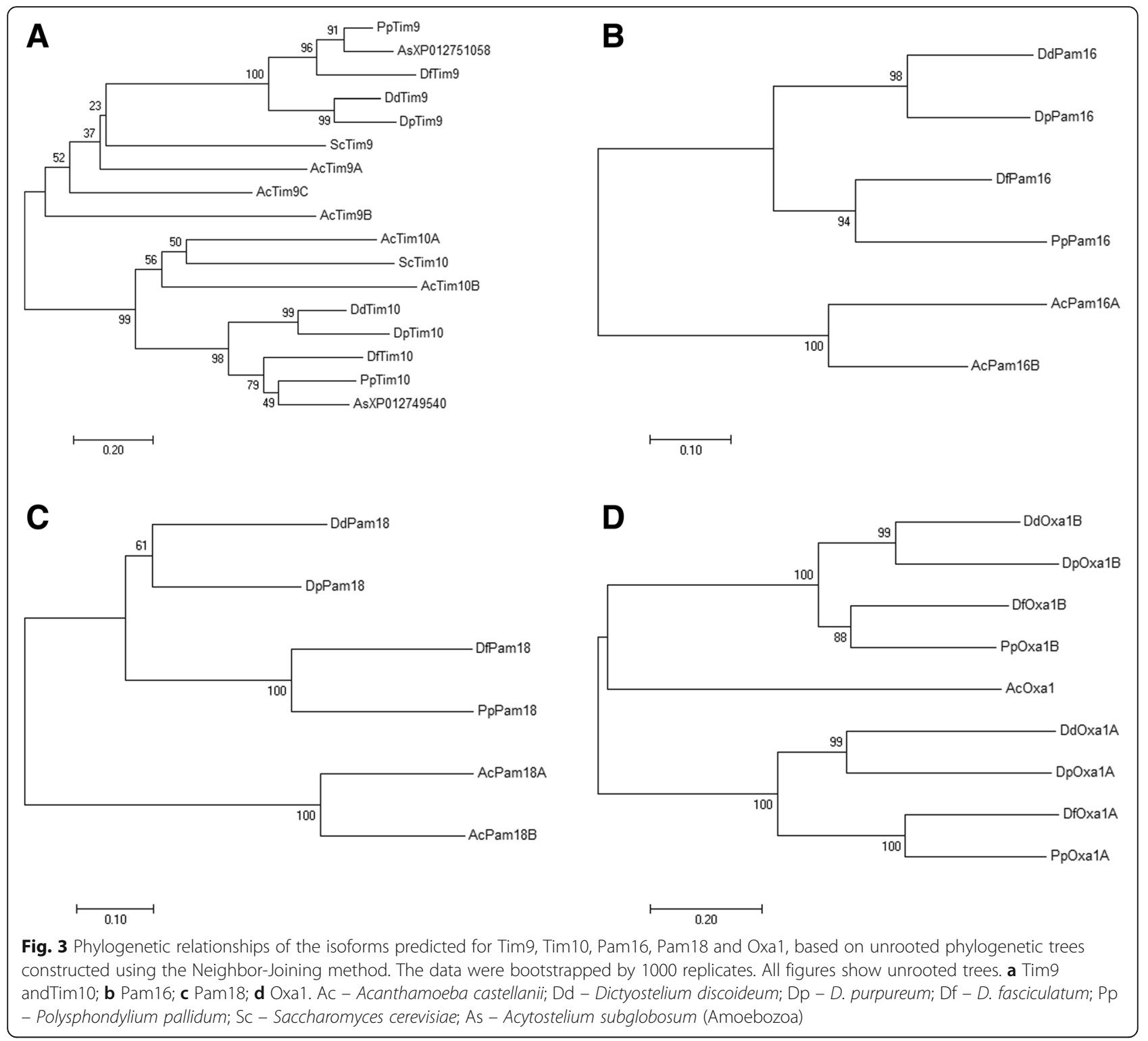

exception of Mia40, the missing subunits are the same as for A. castellanii.

\section{Subunits predicted for entamoebas}

The only subunit predicted for E. dispar and E. nuttalli was $\mathrm{mtHsp70.} \mathrm{The} \mathrm{proteins} \mathrm{have} \mathrm{been} \mathrm{already} \mathrm{annotated}$ (Table 1).

\section{Phylogenetic position of the predicted proteins}

To assess the phylogenetic position of the predicted proteins we estimated the similarity of their amino acid sequences to cognate subunits (Table 2). The predicted subunits displayed mostly the highest similarity to cognate proteins of Opisthokonta (fungi and animals) although the highest similarity to Archaeplastida (plants), Chromalveolata, and Excavata proteins was also observed in some cases. The least diverse in terms of similarity to reference proteins was the MIA complex, as the predicted subunits displayed the highest similarity to Opisthokonta proteins (animals and fungi) with the exception of $D$. fasciculatum Mia40 (Archaeplastida, plants). The prevailing similarity of the predicted subunits to Opisthokonta proteins (animals and fungi) was also observed for TIM23 complex subunits as the similarity to Archaeplastida proteins was only observed for the predicted A. castellanii Tim23 and P. pallidum Tim21. In the case of TIM22 complex as well as Tim9 and Tim 10 proteins, the majority of the predicted proteins also displayed the highest similarity to the cognate Opisthokonta proteins (animals and fungi). However, the highest similarity to Chromalveolata cognate proteins was observed for the predicted D. fasciculatum Sdh3 as 


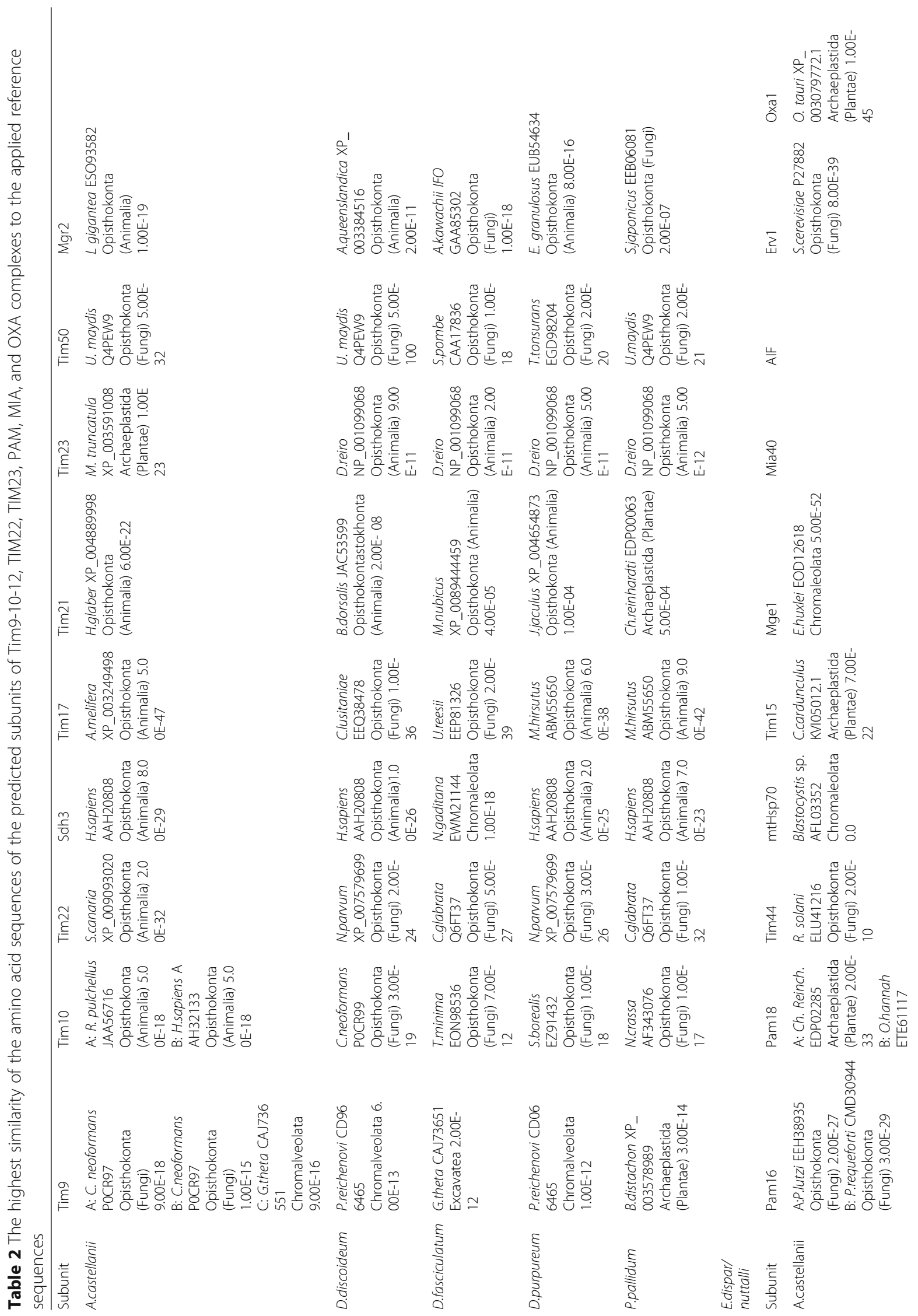




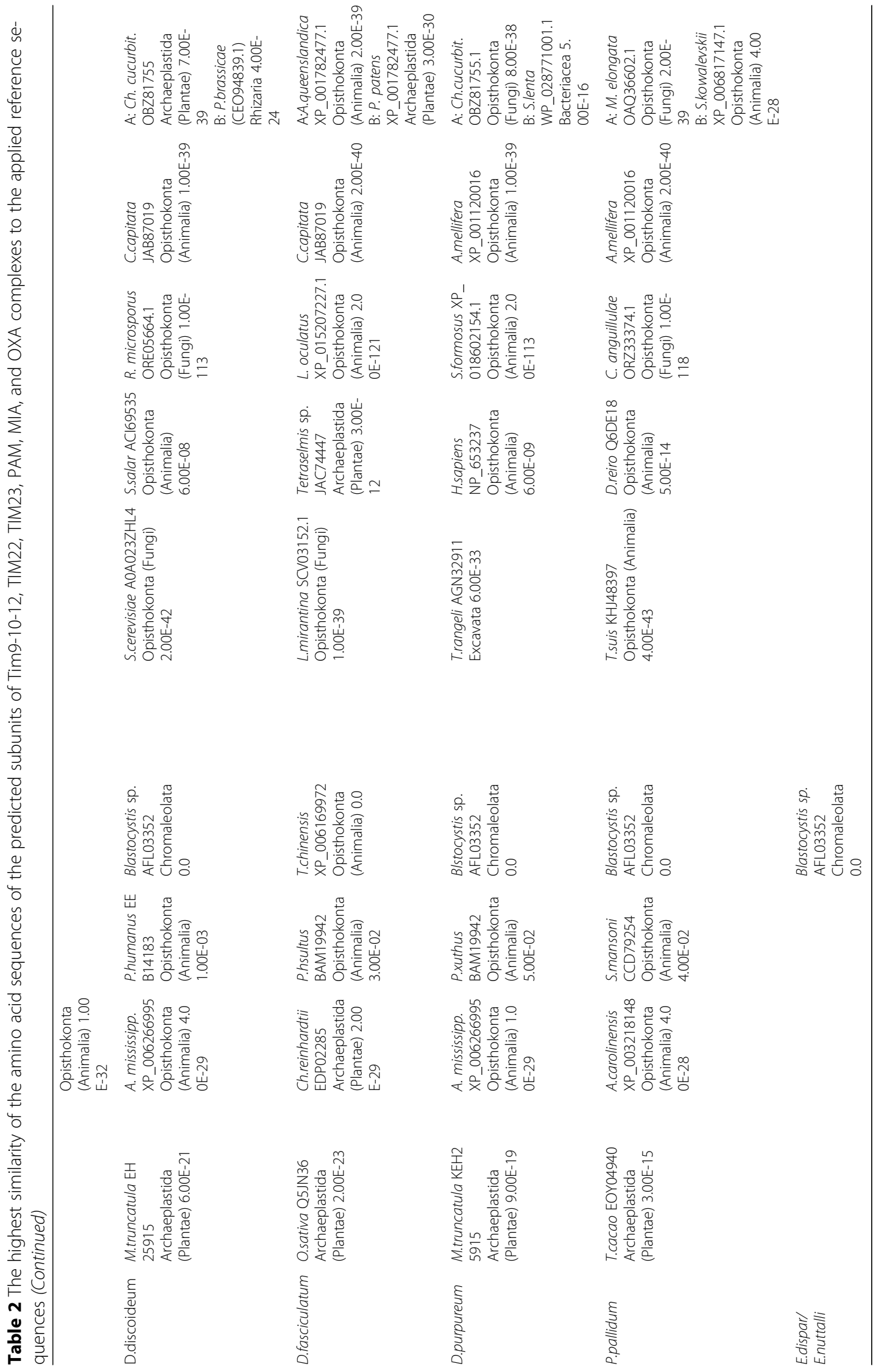


well as A. castellanii Tim9C and the slime mold Tim9 with exception of P. pallidum Tim9, which appeared to be the most similar to the Archaeplastida cognate proteins.

In the case of the predicted subunits of the PAM complex and OXA complex, the phylogenetic origin of proteins regarded as the best match appeared to be more variable. For the PAM complex subunits the similarity concerned the cognate proteins of Opisthokonta (fungi and animals), Archaeplastida, Chromalveolata and Excavata. In the case of the predicted Pam16, the highest similarity was observed for Opisthokonta (fungi) proteins (A. castellanii both isoforms) and Archaeplastida proteins (slime molds). For the predicted Pam18, the highest similarity was observed for Archaeplastida and Opisthokonta (animals) cognate proteins (A. castellanii Pam18A and Pam18B, respectively) whereas the slime mold Pam18 predicted proteins displayed the highest similarity to Opisthokonta (animals) cognate proteins with exception of D. fasciculatum (Archaeplastida). The predicted Tim44 proteins were the most similar to Opisthokonta (fungi) protein (A. castellanii) and Opisthokonta (animal) cognate proteins (all slime molds). Interestingly, all the predicted $\mathrm{mtHsp} 70$ proteins displayed the highest similarity to Chromalveolata cognate proteins, with the exception of $D$. fasciculatum, for which the predicted mtHsp70 appeared to be the most similar to an Opisthokonta (animal) protein. The predicted Mge1 appeared to be the most divergent in regard to phylogenetic relationships, as we observed the best match for Opisthokonta (fungi, animal) proteins (slime molds: D. discoideum, P. pallidum, and D. fasciculatum, respectively), Chromalveolata proteins (A. castellanii), and Excavata (D. purpureum). The same applies to the slime mold predicted Oxa1B isoform that appeared to be a homolog of the predicted A. castellanii Oxa1 (Fig. 3d). The latter was the most similar to an Archaeplastida (plants) cognate protein but the slime mold predicted Oxa1B was the most similar to the cognate proteins of Bacteriaceae, Rhizaria, Archaeplastida (plants), and Opisthokonta (animals), depending on the studied species. However, the second predicted slime mold Oxa1 isoform; i.e. Oxa1A displayed the highest similarity to the Opisthokonta (fungi and animals) and Archaeplastida proteins.

Summing up, the phylogenetic position of the predicted subunits indicated a comparable level of diversity between $A$. castellanii and the slime molds as well as between the slime molds themselves. In the latter, the differences concerning the best match were the most pronounced for D. fasciculatum. However, this observation could not be verified by the involvement of the transcriptome dataset into analysis. Moreover, the obtained results revealed a higher level of diversity in the case of the PAM and OXA complexes. Nevertheless, the predicted subunits were mostly similar in their amino acid sequences to the cognate proteins of Opisthokonta (fungi and animals).

\section{Discussion}

The differences in the subunit organization of the mitochondrial protein import complexes have been mainly reported for members of different phylogenetic lineages, while for members of the same clade the issue is poorly studied. Thus, the data are missing to address the evolutionary aspects of the mitochondrial protein import machinery. As mentioned in the Introduction, the Amoebozoa includes taxa of both biomedical and evolutionary importance. We have recently shown comprehensive analyses of the mitochondrial outer membrane import complexes, i.e. the TOM and TOB/SAM complexes, of members of this supergroup, based on searching of the available genome and transcriptome data [14], but the mitochondrial protein import machinery located in the inner membrane and intermembrane have not been addressed.

Accordingly, the PubMed data searching for the Amoebozoa protein import complexes located in these mitochondrial compartments presents only results of a hidden Markov-model-based analysis performed for the D. discoideum and E. histolytica Tim9-Tim10-Tim12, TIM22, TIM23, and PAM complexes [12, 13, 28]. They predict the presence of the following subunits of the complexes for $D$. discoideum: Tim22 and undefined small Tim proteins (the TIM22 and Tim9-10-12 complexes), Tim17, Tim21, and Tim23 (the TIM23 complex) as well as Pam16, Pam18, and mtHsp70 (the PAM complex), whereas in E. histolytica, only $\mathrm{mtHsp70}$ and a possible homolog of Mge1 have been predicted. Moreover, AIF was identified for $D$. discoideum [29]. Thus, we performed the analysis of genome and transcriptome sequences available for different amoebozoans to obtain a more coherent picture. The analysis includes the Tim9-Tim10-Tim12, TIM22, TIM23, PAM, MIA, and OXA complexes (Fig. 2) of organisms representing different subclades and subdivisions of the Amoebozoa (Fig. 1). In general, all the predicted subunits are conserved between $S$. cerevisiae and the studied amoebozoans.

For E. dispar and E. nuttalli only one subunit of all the studied complexes was predicted, namely $\mathrm{mtHsp70,}$ a member of the PAM complex. It is assumed that all Entamoeba species have mitosomes, although convincing data are still missing (Graham Clark, personal communication). Similarly, in contrast to the well-known pathogenic E. histolytica and E. invadens, the pathogenicity of $E$. dispar and $E$. nuttalli is still a matter of debate [15-17]. Nevertheless, it cannot be concluded that the predicted extreme reduction of the protein import 
apparatus of the mitochondrial inner membrane and the intermembrane space is a marker of a parasitic lifestyle, as parasitic protists are known to express Tim and Pam proteins $[4,13]$. On the other hand, for the studied entamoebas the reduction of the protein import complexes in the inner membrane and intermembrane space seems to be much more pronounced than that of the outer membrane ones, where at least channel-forming subunits are predicted to be present [14]. Accordingly, Tom40 and Tob55/Sam50 as well as Mge1 and mtHsp70 were detected for E. histolytica [13]. However, for other mitosome possessing organisms the higher numbers of the import complex subunits have been predicted, namely Tom55/Sam50, Tom70, Tim17, Tim21, Tim23, Tim22 for Encephalitozoon cuniculi and Tom40, Pam16, Pam18, mtHsp70, Mge1 for Giardia lamblia [13]. Thus, the reduction of the protein import complexes appears to be an indicative feature of mitosomes but it seems that the level of the reduction is multifarious. It should also be remembered that mitosomes may contain proteins that are too divergent to be homologs of known import complex subunits, and consequently cannot be found using only bioinformatic tools [30]. For example, the presence of a novel mitosome outer membrane $\beta$ barrel protein and other two inner membrane proteins of unknown function has been shown to be unique to $E$. histolytica mitosomes [4, 17]. These proteins do not display similarity to canonical import complex subunits. Moreover, the $\beta$-barrel protein named MBOMP30 represents seventh MBOMP subclass lacking any recognizable sequence similarity to any of the six previously identified ones. On the other hand the lack of the transcriptome datasets of $E$. dispar and E. nuttalli may impact the possibility of protein prediction.

For A. castellanii and the slime molds, the organization of the studied complexes is very similar with exception of the MIA complex (Table 1). Moreover, the predicted organization appears to be quite similar to the canonical one described for S. cerevisiae (Fig. 2), Thus it appears that also in these amoebozoan mitochondria the diversity of the inner membrane and the intermembrane space import complexes is less pronounced than in the case of the outer membrane ones [31]. Accordingly, the encoding genes of the slime molds contain similar numbers of predicted exons, being not distinctly different from the numbers predicted for $A$. castellanii putative genes, with the exception of genes encoding Tim50, Mgr2, Tim44, mtHsp70, and Erv1 (Additional file 1: Table S3).

However, a distinct difference is apparent when the predicted subunits of the $A$. castellanii and slime mold Tim9-Tim10-Tim12, PAM, and OXA complexes are compared. Namely, the predicted A. castellanii Tim9, Tim10, Pam16, and Pam18 and the slime mold Oxa1 appear to have isoforms encoded by separate genes. Interestingly, searching of available databases indicates the presence of Tim9, Tim10, and Pam protein isoforms in mitochondria of other organisms, mainly animals and humans [7, 9]. Isoforms were also found for human Tim17 [32] and Tim8 [33-35]. In the case of the OXA complex, two genes encoding Oxa1 and Oxa2 have been reported for Opisthokonta (animals and fungi) and more than two for Archaeplastida (plants) [36]. On the other hand, members of the Excavata seem to have isoforms of Oxa2, while Oxa1 is not present, whereas only one gene encoding Oxa protein and a lack of the protein isoforms have been shown for Chromalveolata [7, 36, 37]. Thus the diversity of the OXA complex organization is still to be explained. Accordingly, the precise roles of the paralogs, including Oxa1 and Oxa2, have yet to be clarified [7]. The difference concerning the presence of small Tims, Pam16, Pam18, and Oxa1 isoforms between the studied amoebozoans constitutes an interesting observation from the point of view of the supergroup phylogeny, as the Amoebozoa are regarded as a sister clade to the Opisthokonta [1, 2]. While the putative expression of the A. castellanii Tim9, Tim10, Pam16, and Pam18 isoforms resembles Opisthokonta cognate proteins, the possible presence of the slime mold Oxal isoforms is much more difficult to explain. Nevertheless, the differences may reflect the evolutionary history of the subclade formation.

As mentioned above, the difference is also observed for the MIA complex as only Erv1 protein is predicted for the A. castellanii MIA complex, whereas Erv1, Mia40 and AIF are predicted for all the studied slime molds. Interestingly, as in the case of plant and mammalian but not S. cerevisiae mitochondria [38], the predicted slime mold Mia40 appears to be a soluble protein (not shown). Therefore, one can suggest that AIF can serve as Mia40 receptor not only in mammalian mitochondria [39-41]. On the other hand, the apparent absence of Mia40 regarded as an essential protein (e.g. [38]) in some eukaryotes has also been reported. For example, the protein does not seem to be present in brown algae [31] and parasitic protists, such as Plasmodium falciparum, Leishmania tarentolae, and Trypanosoma cruzi [42]. Accordingly, A. castellanii is the only proven parasite among the studied amoebozoans, defined as an opportunistic pathogen responsible for amoebic keratitis and granulomatous amoebic encephalitis in humans $[43,44]$. Yet, the conclusions in regard to the correlation between the lack of Mia40 and pathogenicity are at present unjustified. Importantly, the presence of small Tim proteins which undergo oxidative folding, indicate that an oxidative folding machinery exists in $A$. castellanii mitochondria [45]. It suggests that other protein(s) can replace the subunit. Accordingly, for the 
Trypanosoma brucei TOM complex, novel receptors with no homology to known TOM import proteins i.e. ATOM46 and ATOM69, have been identified [46]. Interestingly, these proteins were also found in all kinetoplastids (including the non-pathogenic ones), showing that the expression of the unique proteins is not an adaptation to the parasitic lifestyle of $T$. brucei [47].

Interestingly, amino acids sequence comparison (Table 2) indicates that the predicted subunits of the studied complexes display different levels of amino-acid sequence conservation. The lowest diversity is observed for subunits predicted for the TIM23 and MIA complexes whereas the highest diversity occurs for subunits predicted for the OXA complex, particularly in the case of the slime mold Oxa1B isoform that appears to be a homolog of A. castellanii Oxa1. The differences are not reflected in the encoding gene intron-exon structures (Additional file 1: Table S3), as it has been observed for the outer membrane complexes that excludes different regulation of protein expression at the level of splicing known to support an adaptation to a given lifestyle [14]. Nevertheless, it can be suggested that the different levels of amino acid sequence variability observed for subunits predicted for the inner membrane and intermembrane space import complexes and the outer membrane import complexes may result from natural selection, as the proteins appear to control efficiently organelle biogenesis and function, including both membranes. Accordingly, as it is speculated that differences in amino acid sequences observed between members of different supergroups reflect the early diversification of eukaryotes [47], it can be proposed that within a given supergroup, the variability may reflect emergence of species within its branches.

\section{Conclusions}

Here we present results of a comprehensive bioinformatic analysis of the protein import complexes located in the mitochondrial inner membrane (the TIM22, TIM23, PAM, and OXA complexes) and intermembrane space (the Tim9-Tim10-Tim12 and MIA complexes) of selected species of the Amoebozoa. This analysis was based on searching of the available genome and transcriptome sequences. Our results indicate that in A. castellanii and slime molds, the complex organization appears to be quite similar to the canonical ones described for S. cerevisiae. However, distinct differences are observed in amino acid sequences of some of the predicted proteins and in the numbers of some of the protein isoforms. Moreover, the performed analysis for entamoebas, i.e. E. dispar and E. nuttalli, indicates the absence of any subunit of all the studied complexes except mtHsp70 (the PAM complex), and that further supports the suggestion that all entamoebas have mitosomes. Importantly, the reduction of the protein import apparatus of entamoebas is much more pronounced than in the case of the outer membrane. It seems to be a rule for entamoebas, including $E$. histolytica, but not for all organisms possessing mitosomes. This constitutes an interesting issue from the evolutionary and biomedical perspective, as it addresses the problem of mitochondrial and/or mitosome protein import machinery variability within the currently defined supergroup of eukaryotes. On the other hand, it is becoming clear that the knowledge of mitochondrial protein import of model organisms cannot be generally transferred to all other eukaryotes.

\section{Additional files}

\begin{abstract}
Additional file 1: Table S1. Availability of the genome and transcriptome data of the studied amoebozoans: Acanthamoeba castellanii (AC) [6], Dictyostelium discoideum (Dd) [48], Dictyostelium fasciculatum (Df) [49], Dictyostelium purpureum (Dp) [50] Polysphondylium pallidum (Pp) [48], Entamoeba nuttalli (En) [51], and Entamoeba dispar (Ed) [52]. Table S2. Reference sequences from various eukaryotic lineages for subunits of the small Tims, TIM22, TIM23, PAM, MIA, and OXA complexes obtained by BLAST search. Table S3. Numbers of exons in genes encoding the predicted subunits for the studied complexes of selected Amoebozoa: Acanthamoeba castellanii (A.C), Dictyostelium discoideum (Dd), D. fasciculatum (Df). D. purpureum (Dp), Polysphondylium pallidum (Pp), Entamoeba dispar (Ed), and E. nuttalli (En), compared to selected representatives of fungi, animals, and plants. Figure S1. Alignment of the predicted subunits of the studied complexes displaying differences in amino acid sequences as compared to their counterparts deposited in the GenBank: (A) Acanthamoeba castellanii Tim9A; (B) Polysphondylium pallidum Tim9; (C) A. castellanii Tim10B; (D) A. castellanii Tim22; (E) A. castellanii Tim50; (F) A. castellanii Mgr2; (G) A. castellanii Pam16A; $(H)$ A. castellanii Pam16B; and (I) A. castellanii $\mathrm{mtH}$ sp70. Figure S2. Acanthamoeba castellanii sequences predicted by transcriptome analysis. Amino acid and mRNA assembly correction for the mitochondrial inner membrane and intermembrane space proteins in A. castellanii based on transcriptome and genome assemblies. (DOCX $334 \mathrm{~kb}$ )
\end{abstract}

\section{Abbreviations}

AIF: Apoptosis inducing factor; MIA: Mitochondrial intermembrane space assembly; MIM: Mitochondrial import machinery; OXA: Oxidase assembly factor; PAM: Presequence translocase-associated motor; small Tims: i.e. Tim8Tim13 and Tim9-Tim10-Tim12 intermembrane space complexes; TIM22: Translocase of the inner membrane 22; TIM23: Translocase of the inner membrane 23; TOB/SAM: Topogenesis of the mitochondrial outer membrane $ß$-barrel proteins/sorting and assembly machinery;

TOM: Translocase of the outer membrane

\section{Acknowledgements}

We are grateful to Mateusz Wojtkowski, who helped us to prepare the figures and to Sylwia Ufnalska, who edited the manuscript before submission.

\section{Funding}

This work was supported by the Foundation for Polish Science (MPD Program, grant no. MPD/2010/3) and the Polish Ministry of Science and Higher Education (KNOW RNA Program, grant no.01/KNOW2/2014).

Availability of data and materials

Acanthamoeba castellanii strain Neff used in this study was delivered from American type Culture Collection (ATCC) with the number 30010. 
The datasets generated and analyzed during the current study (alignments and trees) are available in the TreeBASE repository http://purl.org/phylo/ treebase/phylows/study/TB2:S21918

AC RNASeq with the accession number DRA006231 are available in the DDBJ database http://trace.ddbj.nig.ac.jp/BSSearch/ biosample?acc=SAMD00097225

All other data generated and analyzed during this study are included in this published article and its supplementary information files.

\section{Authors' contributions}

HK, MW and WM came up with research ideas. HK and WM supervised the performed analyses. MW, HK and WM wrote the final version of the manuscript. DB carried out the material preparation for the sequencing procedure and bioinformatics analyses. MW and VS performed some of the bioinformatics analyses and MW provided the A. castellanii total RNA. YS conceived the transcriptome sequencing. All authors read and approved the final manuscript.

\section{Ethics approval and consent to participate}

Not applicable.

\section{Consent for publication}

Not applicable.

\section{Competing interests}

The authors declare that they have no competing interests.

\section{Publisher's Note}

Springer Nature remains neutral with regard to jurisdictional claims in published maps and institutional affiliations.

\section{Author details}

${ }^{1}$ Laboratory of Bioenergetics, Institute of Molecular Biology and Biotechnology, Faculty of Biology, Adam Mickiewicz University, Umultowska 89, 61-614 Poznan, Poland. ${ }^{2}$ Institute of Bioinformatics, Faculty of Medicine, University of Muenster, Niels Stensen Strasse 14, 48149 Muenster, Germany. ${ }^{3}$ Department of Medical Genome Sciences, Graduate School of Frontier Sciences, University of Tokyo, Kashiwa, Chiba 277-8562, Japan.

Received: 6 October 2017 Accepted: 14 December 2017

Published online: 29 December 2017

\section{References}

1. Adl SM, Simpson AG, Lane CE, Lukes J, Bass D, Bowser SS, et al. The revised classification of eukaryotes. J Eukaryot Microbiol. 2012;59(5):429-93.

2. Keeling PJ, Burger G, Dyrnford DG, Lang BF, Lee RW, Pearlman RE, Roger AJ, Gray MW. The tree of eukaryotes. Tends Ecol Evol. 2005;20:670-6.

3. Tovar J, Fischer A, Clark CG. The mitosome, a novel organelle related to mitochondria in the amitochondrial parasite Entamoeba histolytica. Mol Microbiol. 1999;32(5):1013-21.

4. Santos HJ, Ima K, Hanadate Y, Fukasawa Y, Oda O, Mi-ichi F, Nozaki T. Screening and discovery of lineage-specific mitosomal membrane proteins in Entamoeba histolytica. Mol Biochem Parasitol. 2016;209(1):10-7.

5. Wojtkowska M, Szczech N, Stobienia O, Jarmuszkiewicz W, Budzinska M, Kmita H. An inception report on the TOM complex of the amoeba Acanthamoeba castellanii, a simple model protozoan in mitochondria studies. J Bioenerg Biomembr. 2005:37(4):261-8.

6. Clarke M, Lohan AJ, Liu B, Lagkouvardos I, Roy S, Zafar N, et al. Genome of Acanthamoeba castellanii highlights extensive lateral gene transfer and early evolution of tyrosine kinase signaling. Genome Biol. 2013;14(2):R11.

7. Neupert W. A perspective on transport of proteins into mitochondria: a myriad of open questions. J Mol Biol. 2015:427(6 Pt A):1135-58.

8. Dolezal P, Likic V, Tachezy J, Lithgow T. Evolution of the molecular machines for protein import into mitochondria. Science. 2006; 313(5785):314-8.

9. Wiedemann N, Pfanner N. Mitochondrial machineries for protein import and assembly. Annu Rev Biochem. 2017;20(86):685-714.

10. Kang Y, Fielden LF, Stojanovski D. Mitochondrial protein transport in health and disease. Semin Cell Dev Biol. 2017.
11. Murcha MW, Wang Y, Narsai R, Whelan J. The plant mitochondrial protein import apparatus - the differences make it interesting. Biochim Biophys Acta. 2014;1840(4):1233-45.

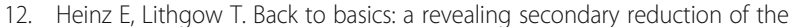
mitochondrial protein import pathway in diverse intracellular parasites. Biochim Biophys Acta. 2013;1833(2):295-303.

13. Makiuchi T, Nozaki T. Highly divergent mitochondrion-related organelles in anaerobic parasitic protozoa. Biochimie. 2014;100:3-17.

14. Buczek D, Wojtkowska M, Suzuki Y, Sonobe S, Nishigami Y, Antoniewicz M, Kmita H, Makałowski W. Protein import complexes in the mitochondrial outer membrane of Amoebozoa representatives. BMC Genomics. 2016;17:99.

15. Stairs CW, Leger MM, Roger AJ. Diversity and origins of anaerobic metabolism in mitochondria and related organelles. Philos Trans R Soc Lond Ser B Biol Sci. 2015;370(1678):20140326.

16. Ackers JP. The diagnostic implications of the separation of Entamoeba histolytica and Entamoeba dispar. J Biosci. 2002;27:573-8.

17. Santos HJ, Imai K, Makiuchi T, Tomii K, Horton P, Nozawa A, Ibrahim M, Tozawa Y, Nozaki T. A novel mitosomal $\beta$-barrel outer membrane protein in entamoeba. Sci Rep. 2015:5:8545.

18. Neff RJ. Purification, axenic cultivation, and description of a soil amoeba, Acanthamoeba sp. J Protozool. 1957:4:176-82.

19. Grabherr MG, Haas BJ, Yassour M, Levin JZ, Thompson DA, Amit I, et al. Fulllength transcriptome assembly from RNA-Seq data without a reference genome. Nat Biotechnol. 2011;29(7):644-52.

20. Altschul SF, Gish W, Miller W, Myers EW, Lipman DJ. Basic local alignment search tool. J Mol Biol. 1990;215(3):403-10.

21. Eddy SR. Accelerated profile HMM searches. PLoS Comput Biol. 2011;7(10):e1002195.

22. Artimo P, Jonnalagedda M, Arnold K, Baratin D, Csardi G, de Castro E, et al. ExPASy: SIB bioinformatics resource portal. Nucleic Acids Res. 2012;40(Web Server issue):W597-603.

23. Thompson JD, Higgins DG, Gibson TJ. CLUSTAL W: improving the sensitivity of progressive multiple sequence alignment through sequence weighting, position-specific gap penalties and weight matrix choice. Nucleic Acids Res. 1994:22(22):4673-80

24. Saitou N, Nei M. The neighbor-joining method: a new method for reconstructing phylogenetic trees. Mol Biol Evol. 1987:4(4):406-25.

25. Tamura K, Peterson D, Peterson N, Stecher G, Nei M, Kumar S. MEGA5: molecular evolutionary genetics analysis using maximum likelihood, evolutionary distance, and maximum parsimony methods. Mol Biol Evol. 2011;28(10):2731-9.

26. Kumar S, Stecher G, Tamura K. MEGA7: molecular evolutionary genetics analysis version 7.0 for bigger datasets. Mol Biol Evol. 2016;33:1870-4.

27. Felsenstein J. Confidence limits on phylogenies: an approach using the bootstrap. Evolution. 1985;39:783-91.

28. Dolezal P, Dagley MJ, Kono M, Wolynec P, Likic VA, Foo JH, Sedinova H, Tachezy J, Bachmann A, Bruchhaus I, Lithgow T. The essentials of protein import in the degenerate mitochondrion of Entamoeba histolytica. PLoS Pathog. 2010;6(3):e1000812.

29. Kadam AA, Jubin T, Mir HA, Begum R. Potential role of apoptosis inducing factor in evolutionarily significant eukaryote, Dictyostelium discoideum survival. Biochim Biophys Acta. 2017;1861(1 Pt A):2942-55.

30. Likic VA, Dolezal P, Celik N, Dagley M, Lithgow T. Using hidden markov models to discover new protein transport machines. Methods Mol Biol. 2010;619:271-84.

31. Carrie C, Murcha MW, Whelan J. An in silico analysis of the mitochondrial protein import apparatus of plants. BMC Plant Biol. 2010;10:249.

32. Rainbolt TK, Atanassova N, Genereux JC, Wiseman RL. Stress-regulated translational attenuation adapts mitochondrial protein import through Tim17A degradation. Cell Metab. 2013;18(6):908-19.

33. Sokol AM, Sztolsztener ME, Wasilewski M, Heinz E, Chacinska A. Mitochondrial protein translocases for survival and wellbeing. FEBS Lett. 2014:588(15):2484-95.

34. Hennon SW, Soman R, Zhu L, Dalbey RE. YidC/Alb3/Oxa1 family of Insertases. J Biol Chem. 2015:290(24):14866-74.

35. Stiller SB, Höpker J, Oeljeklaus S, Schütze C, Schrempp SG, Vent-Schmidt J, Horvath SE, Frazier AE, Gebert N, van der Laan M, Bohnert M, Warscheid B, Pfanner N, Wiedemann N. Mitochondrial OXA translocase plays a major role in biogenesis of inner-membrane proteins. Cell Metab. 2016;23(5):901-8.

36. Benz M, Soll J, Ankele E. Arabidopsis thaliana Oxa proteins locate to mitochondria and fulfill essential roles during embryo development. Planta. 2013;237:573-88. 
37. Zhang YJ, Tian HF, Wen JF. The evolution of YidC/Oxa/Alb3 family in the three domains of life: a phylogenomic analysis. BMC Evol Biol. 2009;9:137.

38. Chacinska A, Koehler CM, Milenkovic D, Lithgow T, Pfanner N. Importing mitochondrial proteins: machineries and mechanisms. Cell. 2009;138(4):628-44.

39. Hangen E, Féraud O, Lachkar S, Mou H, Doti N, Fimia GM, et al. Interaction between AIF and CHCHD4 regulates respiratory chain biogenesis. Mol Cell. 2015 Jun 18;58(6):1001-14.

40. Meyer K, Buettner S, Ghezzi D, Zeviani M, Bano D, Nicotera P. Loss of apoptosis-inducing factor critically affects MIA40 function. Cell Death Dis. 2015;6:e1814.

41. Modjtahedi N, Kroemer G. CHCHD4 links AlF to the biogenesis of respiratory chain complex I. Mol Cell Oncol. 2015;3(2):e1074332.

42. Eckers E, Petrungaro C, Gross D, Riemer J, Hell K, Deponte M. Divergent molecular evolution of the mitochondrial sulfhydryl:cytochrome $C$ oxidoreductase Erv in opisthokonts and parasitic protists. J Biol Chem. 2013; 288(4):2676-88.

43. Saheb E, Trzyna W, Bush J. Caspase-like proteins: Acanthamoeba castellanii metacaspase and Dictyostelium discoideum paracaspase, what are their functions? J Biosci. 2014;39(5):909-16.

44. Leger MM, Gawryluk RMR, Gray MW, Roger AJ. Evidence for a hydrogenosomal-type anaerobic ATP generation pathway in Acanthamoeba castellanii. PLoS One. 2013:8(9):e69532.

45. Stojanowski D, Bragoszewski P, Chacińska A. The MIA pathway: a tight bond between protein transport and oxidative folding in mitochondria. Biochim Biophys Acta. 2012;1823(7):1142-50.

46. Mani J, Desy S, Niemann M, Chanfon A, Oeljeklaus S, Pusnik M, Schmidt O, Gerbeth C, Meisinger C, Warscheid B, Schneider A. Mitochondrial protein import receptors in Kinetoplastids reveal convergent evolution over large phylogenetic distances. Nat Commun. 2015;6:6646.

47. Mani J, Meisinger C, Schneider A. Peeping at TOMs-diverse entry gates to mitochondria provide insights into the evolution of eukaryotes. Mol Biol Evol. 2016;33(2):337-51.

48. Eichinger L, Pachebat JA, Glockner G, Rajandream MA, Sucgang R, Berriman $\mathrm{M}$, et al. The genome of the social amoeba Dictyostelium discoideum. Nature. 2005:435(7038):43-57.

49. Heidel AJ, Lawal LH, Felder M, Schilde C, Helps NR, Tunggal B, Rivero F, John U, Schleicher M, Eichinger L, Platzer M, Noegel AA, Schaap P, Glockner G. Phylogeny-wide analysis of social amoeba genomes highlights ancient origins for complex intercellular communication. Genome Res. 2011:21(11): 1882-91.

50. Sucgang R, Kuo A, Tian X, Salerno W, Parikh A, Feasley CL, et al. Comparative genomics of the social amoebae Dictyostelium discoideum and Dictyostelium purpureum. Genome Biol. 2011;12(2):R20.

51. Tachibana H, Yanagi T, Pandey K, Cheng XJ, Kobayashi S, Sherchand JB, Kanbara H. An Entamoeba sp. strain isolated from rhesus monkey is virulent but genetically different from Entamoeba histolytica. Mol Biochem Parasitol. 2007;153(2):107-14.

52. Wang Z, Samuelson J, Clark CG, Eichinger D, Paul J, Van Dellen K, Hall N, Anderson I, Loftus B. Gene discovery in the Entamoeba invadens genome. Mol Biochem Parasitol. 2003;129(1):23-31.

\section{Submit your next manuscript to BioMed Central and we will help you at every step:}

- We accept pre-submission inquiries

- Our selector tool helps you to find the most relevant journal

- We provide round the clock customer support

- Convenient online submission

- Thorough peer review

- Inclusion in PubMed and all major indexing services

- Maximum visibility for your research

Submit your manuscript at www.biomedcentral.com/submit 